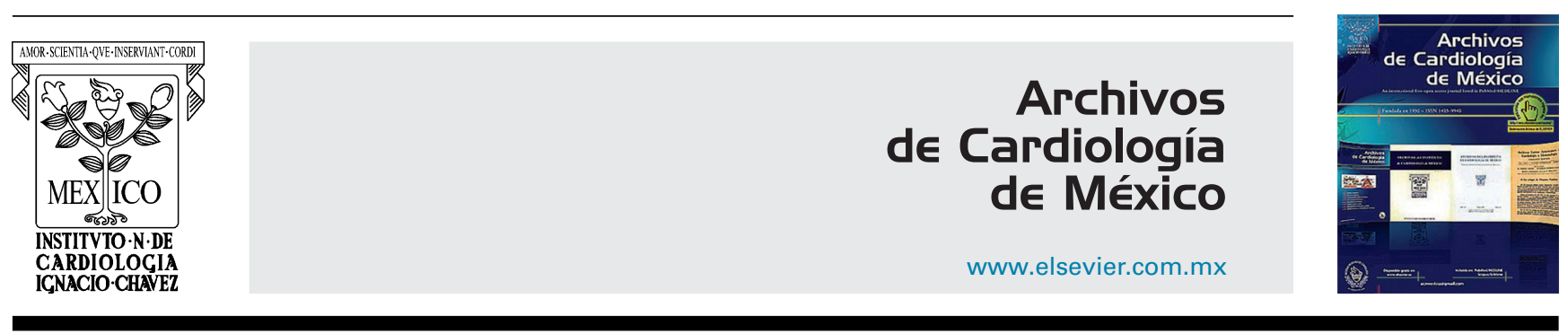

ARTÍCULO DE REVISIÓN

\title{
Peculiaridades de la ecocardiografía transesofágica en pacientes octogenarios
}

\author{
Jesús M. Canale ${ }^{a, *}$, Rubén Cardoza-Encinas ${ }^{\mathrm{b}}$ y Andrés Canale-Segovia ${ }^{\mathrm{c}}$ \\ a Instituto del Corazón (INCOR), Hospital CIMA, Hermosillo, Sonora, México \\ b Unidad de Terapia Intensiva, Hospital CIMA, Hermosillo, Sonora, México \\ c Departamento de Medicina y Ciencias de la Salud, Universidad de Sonora, Hermosillo, Sonora, México
}

Recibido el 27 de abril de 2016; aceptado el 14 de noviembre de 2016

\section{PALABRAS CLAVE \\ Ecocardiografía transesofágica; Adulto mayor; Octogenario; México}

\section{KEYWORDS}

Transesophageal echocardiography; Elderly; Octogenarian; Mexico

\begin{abstract}
Resumen La tendencia creciente en el número absoluto y relativo de ancianos en la composición demográfica, el incremento en la prevalencia de cardiopatías conforme se avanza en edad y el impresionante desarrollo tecnológico de los ultrasonógrafos son factores que han impulsado recientemente un mayor número de ecocardiogramas transesofágicos en pacientes de edad muy avanzada, sin embargo la información publicada sobre las características distintivas del método en el paciente anciano es aún escasa y muy probablemente insuficiente. Esta revisión se enfoca en material bibliográfico publicado sobre las características más peculiares -tanto metodológicas como diagnósticas- de la ecocardiografía transesofágica en el paciente octogenario o aún mayor, a fin de presentarlas aquí de una manera ordenada y sistematizada de tal forma que aporten elementos para fomentar un criterio clínico útil en el médico a cargo de pacientes de esa franja etaria en quienes esta modalidad diagnóstica es cada vez más requerida.

( 2016 Instituto Nacional de Cardiología Ignacio Chávez. Publicado por Masson Doyma México S.A. Este es un artículo Open Access bajo la licencia CC BY-NC-ND (http://creativecommons. org/licenses/by-nc-nd/4.0/).
\end{abstract}

\section{Peculiarities of the transesophageal echocardiography in octogenarian patients}

Abstract The growing trend in the absolute and relative number of elderly in the population scheme, the increasing prevalence of heart disease as people get older and the impressive technological development of ultrasound devices have all together recently contributed to promote an increasing number of transesophageal echocardiograms in older patients, however, the already published information about the distinguishing features of this diagnostic methodology in elderly patients is still scarce and seems to be insufficient. This review focuses on the already published methodological and diagnostic features related to the transesophageal echocardiography in the octogenarian -or even older patient- in order to show them in an orderly and

\footnotetext{
* Autor para correspondencia. Hospital CIMA-INCOR, Paseo Río San Miguel 35, Colonia Proyecto Río Sonora, Hermosillo, Sonora, México. 83280.

Correo electrónico: jesus.canale@gmail.com (J.M. Canale).
} 
systematic manner to provide elements that induce useful clinical criteria for the physician who attends patients in this age group in whom this diagnostic modality is now increasingly requested.

(C) 2016 Instituto Nacional de Cardiología Ignacio Chávez. Published by Masson Doyma México S.A. This is an open access article under the CC BY-NC-ND license (http://creativecommons. org/licenses/by-nc-nd/4.0/).

\section{Introducción}

La metodología procedimental de la ecocardiografía transesofágica (ETE) se encuentra referida en múltiples citas pero son pocas y quizás insuficientes las que se enfocan de manera específica a las peculiaridades de este método para el adulto mayor. Tomando en cuenta el incremento absoluto y proporcional de la población de ancianos a nivel global, y en consecuencia el número creciente de adultos mayores que se estudian hoy con ETE, se percibe conveniente investigar en la literatura médica indexada las peculiaridades que caracterizan la ETE en el paciente añoso, y más especialmente al de 80 y más años de edad, referentes tanto a la preparación y desarrollo del procedimiento en sí como también a las peculiaridades diagnósticas, es decir, sobre aquellos hallazgos que son más característicos en los pacientes de este grupo etario. Partimos de la hipótesis de que la preparación para el procedimiento, la realización del mismo, así como los hallazgos que son más comunes en el anciano difieren de los que caracterizan la ETE en pacientes de otras franjas etarias. Más allá de la tarea meramente descriptiva de tales características, nos hemos abocado también a proponer algunas adecuaciones pertinentes para la planeación y realización personalizada de esta modalidad diagnóstica en el octogenario.

\section{Métodos}

Este es un estudio de revisión de datos publicados en la literatura médica relativa a las características más propias -aunque no totalmente exclusivas- de personas de 80 y más años de edad que supondrían una diferencia útil de ser tomada en consideración al momento de planear, preparar, efectuar e interpretar un estudio de ETE en el adulto mayor. La revisión se efectuó a partir de aquellas peculiaridades, trastornos y/o enfermedades en cierto modo más característicos de la senectud y que podrían motivar un compromiso en el personal sanitario para tomar previsiones y precauciones especiales para efectuar el procedimiento así como aquellas características que se refieren a los hallazgos más peculiares en razón propia de la edad avanzada e igualmente los cuidados a observar después de un estudio de ETE, especialmente en la fase de vigilancia inmediata posprocedimiento.

\section{Resultados}

Para mayor claridad consideremos los resultados (tablas 1 y 2) de la investigación bibliográfica en 2 vertientes: primeramente, las limitaciones o comorbilidades más peculiares del
Tabla 1 Comorbilidades más relevantes a considerar en pacientes octogenarios candidatos a ecocardiograma transesofágico

Deterioro cognitivo
Déficit visual
Déficit auditivo
Vértigo
Osteoartropatías degenerativas
Enfermedad de Parkinson
Hiperplasa prostática benigna
Incontinencia urinaria
Neumopatía (sobredistensión pulmonar), hipoventilación
Glaucoma
Enfermedad esofágica
Polifarmacia e interacciones medicamentosas

octogenario que pudieran tener implicaciones en la preparación y en el desarrollo de un estudio de ETE, y, más adelante, los hallazgos anatómicos y/o patológicos más prevalecientes en la ETE del octogenario y que podrían igualmente constituir una peculiaridad distintiva, aunque no exclusiva, de su edad. Todo paciente que se propone para un procedimiento médico tiene derecho a estar bien informado de las características e implicaciones de este de manera personalizada para que pueda aceptarlo o rechazarlo legítima y auténticamente, y para este propósito es necesario que su estado cognitivo sea adecuado. Conforme se avanza de la edad adulta a la senectud el estado cognitivo va sujeto

Tabla 2 Condiciones, hallazgos o trastornos cardiovasculares más peculiares del paciente senil en relación con la ecocardiografía transesofágica

Implicación embolígena de la fibrilación auricular: trombosis de la orejuela y/o de la aurícula izquierda Degeneración calcificante de la válvula aórtica Degeneración calcificante de la válvula mitral Aterosclerosis del arco aórtico Aneurisma de la aorta torácica

Probable candidato a intervencionismo estructural Para implante valvular aórtico transcatéter Para implante de un dispositivo de cierre de la orejuela izquierda

Malformaciones cardiacas con sobrevida prolongada (en especial la válvula aórtica bicúspide y el cortocircuito a nivel auricular) 
a más probabilidades de deterioro ${ }^{1}$; en este sentido, un estudio conducido por Bermejo-Pareja et al. mostró que la incidencia de diversos subtipos de demencia tiene una tasa de 9 nuevos casos por cada 1,000 personas/año entre los 64 y 74 años de edad mientras que de los 80 a los 89 años de edad muestra una tasa de 63, 7 veces mayor ${ }^{2}$. El déficit cognitivo entorpece no solo el entendimiento del procedimiento por parte del paciente sino también la cooperación con la que él pudiera participar en el curso del estudio. El enfermo afectado por la enfermedad de Alzheimer -una de las modalidades demenciales más frecuentes en el adulto mayor- puede manifestar desde fallas de memoria y momentos de confusión, especialmente cuando no está en su entorno familiar, hasta desorientación, dificultad para expresarse a través del habla, inquietud y paranoia ${ }^{3}$. Este problema no es menor toda vez que en el año 2010 se estimó que había 35 millones de personas con demencia en el mundo y se advierte que tal cifra se duplicará cada 20 años alcanzando a más de 65 millones para el año $2030^{4}$. Aun conservando un adecuado estado cognitivo, el octogenario podrá tener dificultad para asimilar cabalmente las explicaciones sobre el método debido a un déficit visual ${ }^{5} 0$ auditivo $^{6}$ especialmente cuando en el laboratorio de ETE en cuestión se acostumbre hacer una explicación previa al estudio mediante ayudas audiovisuales como láminas, vídeos, etcétera. Por otra parte, el vértigo no solo es más frecuente conforme se avanza en edad sino que también va siendo el resultado de un número cada vez mayor de causas en el anciano; una de las modalidades más reconocidas de este trastorno es el vértigo postural paroxístico que se incluye en los trastornos del equilibrio corporal y que si bien a los 70 años de edad tiene una prevalencia del $32 \%$, entre los 88 y 90 años de edad, su tasa se eleva al $52 \%$. Si en el paciente se desencadena un episodio de vértigo vinculado a la posición corporal que de ordinario sea la más adecuada para su ETE, esto generará alteraciones en el desarrollo del procedimiento debido a los síntomas de inquietud, sensación de desbalance o desequilibrio corporal, náuseas y diaforesis, signos y síntomas que, además, podrían confundir al equipo sanitario que le asiste pues pueden inducirle a suponer que está ocurriendo alguna complicación de otro género. Las alteraciones musculoesqueléticas pueden ser más frecuentes en el octogenario y suponen mayor dificultad para guardar la mejor postura corporal durante la ETE, como es el caso de la discartrosis cervical, la xifosis o hiperxifosis dorsal cuya prevalencia ciertamente es mayor en el anciano ${ }^{8}$. Situación similar puede observarse en los casos de fractura de cadera, condición que también suele guardar relación con la edad avanzada y más aún en octogenarios ${ }^{9}$. La enfermedad de Parkinson, que es poco común antes de los 50 años de edad, incrementa su incidencia y prevalencia en los grupos de mayor edad, hasta la novena década de la vida ${ }^{10,11}$ y sus manifestaciones locomotoras como el temblor, la bradicinesia y la rigidez muscular ${ }^{12}$ podrían interferir la adopción de una posición corporal cómoda para el paciente y en consecuencia interferir su capacidad o disposición efectiva para cooperar adecuadamente durante el estudio. Otro problema a considerar es el caso de la hiperplasia prostática, condición cuya prevalencia aumenta más a mayor edad del adulto: mientras su prevalencia es del $2.7 \%$ en hombres con edades de 45 a 49 años, a la edad de 80 años la prevalencia se dispara tanto como al $24 \%$, es decir, en uno de cada 4 octogenarios ${ }^{13}$, en los que el titubeo o «vacilación» urinaria, frecuente en ese trastorno, puede evitar un adecuado vaciamiento vesical en la fase previa a la ETE ocasionándole molestias en el curso del estudio como son los episodios de urgencia miccional e incluso incontinencia urinaria y/o molestia suprapúbica durante el procedimiento con significativos inconvenientes para continuar con una razonable normalidad el estudio. En el caso de la mujer, mientras la incontinencia urinaria en la adulta joven muestra una prevalencia del $20 \%$ al $30 \%$, en la mujer de edad media alcanza entre el $30 \%$ y el $40 \%$, pero en la anciana tiene una prevalencia hasta del $50 \%{ }^{14}$, factor que por razones lógicas debe tomarse en cuenta en previsión del procedimiento. Ahora bien, si se planea utilizar sedación, como ocurre en la mayoría de los procedimientos de ETE en muchos centros, habrá que considerar que la hipoventilación y la hipoxemia se presentan más fácilmente en los ancianos ${ }^{15}$, lo cual supondrá -por ejemplo, y entre otros riesgos- mayor probabilidad de afectación miocárdica con generación de arritmias, gasto contráctil o isquemia. La Agencia Nacional de Seguridad del Paciente del Reino Unido (NPSA, por sus siglas en inglés) emitió en el año 2009 una advertencia referente a la mayor probabilidad de eventos adversos en ancianos que reciben midazolam y se ha sugerido que en el paciente geriátrico se inicie con un bolo a baja dosis: $1 \mathrm{mg}$ intravenoso ${ }^{16}$. Es útil conocer que el anciano durante una ETE tiene un riesgo 3.5 veces mayor de desarrollar hipotensión arterial transitoria que el riesgo de un paciente adulto joven ${ }^{17}$. En la eventualidad de que un anciano presentase reacción vasovagal y se plantee manejarle con atropina, el equipo a su cargo deberá conocer si el paciente ha sido diagnosticado de padecer glaucoma, y en caso tal, tomar las precauciones pertinentes para evitar desafortunadas complicaciones oculares ${ }^{18,19}$. Diversos trastornos estructurales o funcionales del esófago pueden complicar un estudio de ETE y en este sentido téngase como dato suficiente que el cáncer de esófago es 20 veces más frecuente en personas mayores de 65 años de edad que en sujetos de menor edad ${ }^{20}$ y tómese como ejemplo relevante para la experiencia el de un caso de perforación esofágica en un adulto mayor durante el monitoreo transoperatorio con $E T E$, complicación trágica que se atribuyó a isquemia por compresión de tejido esofágico lábil ${ }^{21}$. En este tema debe tomarse nota de que los ancianos con acalasia del esófago, según lo observado en un estudio de Clouse et al., tienen una reducción de la elevación de la presión residual posdeglucional del esfínter esofágico inferior y sufren menos dolor torácico anterior que los pacientes de menor edad, hecho que podría en cierta medida enmascarar el diagnóstico y pasarse por alto la detección oportuna de acalasia en el octogenario antes de llegar a la ETE ${ }^{22}$. Finalmente, es un hecho de observación bien conocido que con frecuencia los adultos mayores reciben múltiples fármacos ${ }^{23}$ y es muy importante identificarlos antes del procedimiento con el objeto de prever posibles interacciones con los medicamentos planeados para la sedación o para otros propósitos.

Ahora bien, en cuanto a los hallazgos anatómicos o patológicos que suponen cierta peculiaridad diagnóstica de la ETE en los octogenarios y mayores, mencionemos en primer término el corazón embolígeno a partir de la fibrilación auricular, arritmia cuya prevalencia se reportó del $10 \%$ al pasar los 80 años de edad según los datos de una serie publicada en el año $2001^{24}$; sin embargo, en una amplia serie publicada en 
el año 2014 sobre población residente en España se encontró una prevalencia del $17.7 \%$ para esa arritmia en personas igualmente de 80 y más años de edad ${ }^{25}$, por cierto, franja etaria en la que también otros factores que elevan la probabilidad de embolización sistémica son más prevalecientes: hipertensión arterial, insuficiencia cardiaca, enfermedad arterial periférica, diabetes mellitus y antecedente de tromboembolismo sistémico. Otro hallazgo de riesgo embolígeno y de prevalencia creciente en el anciano es la ateroesclerosis del arco aórtico que hoy se considera un factor de riesgo mayor e independiente para embolismo cerebral ${ }^{26,27}$. La calcificación del anillo mitral es un proceso degenerativo de observación más frecuente en el adulto mayor ${ }^{28}$ y se ha percibido que en el anciano existe una asociación entre aquella y el doble de riesgo de sufrir un evento vascular cerebral ${ }^{29}$. La calcificación de válvula aórtica tricúspide es un hallazgo relativamente común a edades avanzadas, con un pico en la octava y novena décadas de la vida ${ }^{30}$ y con relación a este punto un documento de la Asociación Europea de Ecocardiografía clasifica la estenosis aórtica calcificada como de riesgo menor o incierto de fuente embolígena ${ }^{31}$. La identificación de un aneurisma de la aorta torácica es de especial interés a edades avanzadas, quizás más en mujeres, toda vez que se observó que la incidencia ajustada para edad y sexo de esta dolencia en un estudio comunitario basado en la población fue de 5.9 nuevos aneurismas por 100,000 personas/año tras un periodo de observación de 30 años, con una mediana de edades de 65 años para el varón en tanto que para la mujer fue de 77 años ${ }^{32}$, factores todos estos a considerar toda vez que en el estudio de esta dolencia la ETE guarda un papel bien definido. En relación con la ETE para la selección de pacientes que pudieran ser candidatos a intervencionismo estructural, una revisión del material correspondiente de 34 centros reveló que para el implante de válvula aórtica transcatéter la edad media al tiempo de la inclusión fue de 82.7 años $^{33}$, lo que indica obviamente que este procedimiento es por ahora un recurso para el manejo de un problema con mayor prevalencia en el octogenario. De igual manera, la ETE es muy útil tanto para guiar la oclusión de la orejuela izquierda por abordaje percutáneo como para su seguimiento, tratándose de un procedimiento intervencionista de creciente utilización en el paciente senil con fibrilación auricular para reducir el riesgo de sangrado que representa la terapia anticoagulante ${ }^{34}$. Finalmente, cabe mencionar que en el octogenario las probabilidades de encontrar malformaciones cardiacas complejas en ETE son notablemente menores que a edades más tempranas aunque es verdad que la tendencia a identificar malformaciones cardiacas en el adulto va en ascenso, pues baste considerar que, de los nacidos en 1950 con malformación cardiaca, cuando más el $20 \%$ llegaba a la vida adulta en tanto que hoy se estima que hasta un $90 \%$ lo está logrando ${ }^{35}$. Los tipos más comunes de cardiopatía congénita encontrada en el adulto mayor son los cortocircuitos (60\%) y las valvulopatías (37\%); los defectos severos constituyen solamente el $3 \%^{36}$.

\section{Discusión}

Ciertamente la mayoría de los hallazgos relevantes para un diagnóstico pueden identificarse mediante un ecocardiograma transtorácico pero con alguna frecuencia esto no es posible. Es el caso, entre otros, cuando existen condiciones anatómicas que interfieren con la propagación adecuada del haz ultrasónico como suele ocurrir en algunos casos de neumopatía -especialmente con sobredistensión pulmonarantecedente de implante de prótesis mamaria o quemaduras de la piel de la cara anterior del tórax. En la figura 1 se muestra la mejor imagen posible que fue obtenida por ecocardiograma transtorácico en un varón adulto mayor neumópata y que resultó insuficiente para precisar el diagnóstico y orientar las alternativas de manejo, e igualmente se presentan las imágenes obtenidas mediante ETE bidimensional y tridimensional con las que se logró establecer un diagnóstico preciso y de utilidad definitiva para la planeación del manejo. En otras ocasiones la necesidad de un ETE radicará en alguna condición que difícilmente puede identificarse por ecocardiograma transtorácico, como la presencia de trombos en la orejuela izquierda, el sitio y extensión de la disección de un aneurisma aórtico o la demostración de la permeabilidad de un foramen ovale; otras veces la ETE será necesaria para guiar y/o darle seguimiento a algún procedimiento de intervencionismo estructural percutáneo, algunos de creciente demanda en el adulto mayor como el implante transcatéter de válvula aórtica y el cierre de la orejuela izquierda mediante la implantación de un dispositivo de oclusión.

Para tener la certeza de que el paciente octogenario programado para una ETE comprenda cabalmente en qué consiste el procedimiento y esté realmente bien informado de sus características, beneficios, riesgos y alternativas -como es su derecho legal y además un deber ético del examinador- es necesario hacer una ágil valoración de su estado cognitivo así como del estado de sus capacidades auditiva y visual. Esto puede lograrse de manera suficiente con una breve conversación inicial por parte del personal que le asistirá en el estudio. Existen diversas pruebas clínicas para la evaluación del deterioro cognitivo, algunas de ellas son breves y útiles para una valoración ágil incluso a nivel de atención primaria. ${ }^{37} \mathrm{Si}$ se identifica que el paciente no está en condiciones de asimilar bien la información conviene entonces explicar el procedimiento de manera pausada, sencilla y concreta ayudándose quizás con expresiones verbales y corporales adecuadas por parte de quien dé la explicación. Cuando aun así quedara muy claro que su capacidad cognitiva no permitirá una asimilación suficiente de la información, habrá que proporcionarla entonces al familiar o al responsable del cuidado del anciano, de conformidad con las disposiciones legales de la región y los estándares bioéticos del establecimiento. Por otra parte, en caso de que se advierta alguna dificultad motora, ya sea de origen neurológico o musculoesquelético, debe preguntarse al paciente o a su cuidador sobre la posibilidad de una postura adecuada para el paciente y es muy recomendable que el personal del gabinete tenga previstos aditamentos y posturas corporales alternativas, especialmente en los casos de xifosis dorsal acentuada o artropatía cervical, condiciones que son relativamente frecuentes. Cuando se trata de un paciente con xifosis y el estudio se hará en decúbito dorsal -por ejemplo, durante intervencionismo estructural o en algún caso de vértigo postural o en pacientes en estado crítico- se valorará la colocación de material de elevación suficiente y cómodo sobre el que descansará la cabeza. En la figura 2 se presentan imágenes de una paciente de 84 

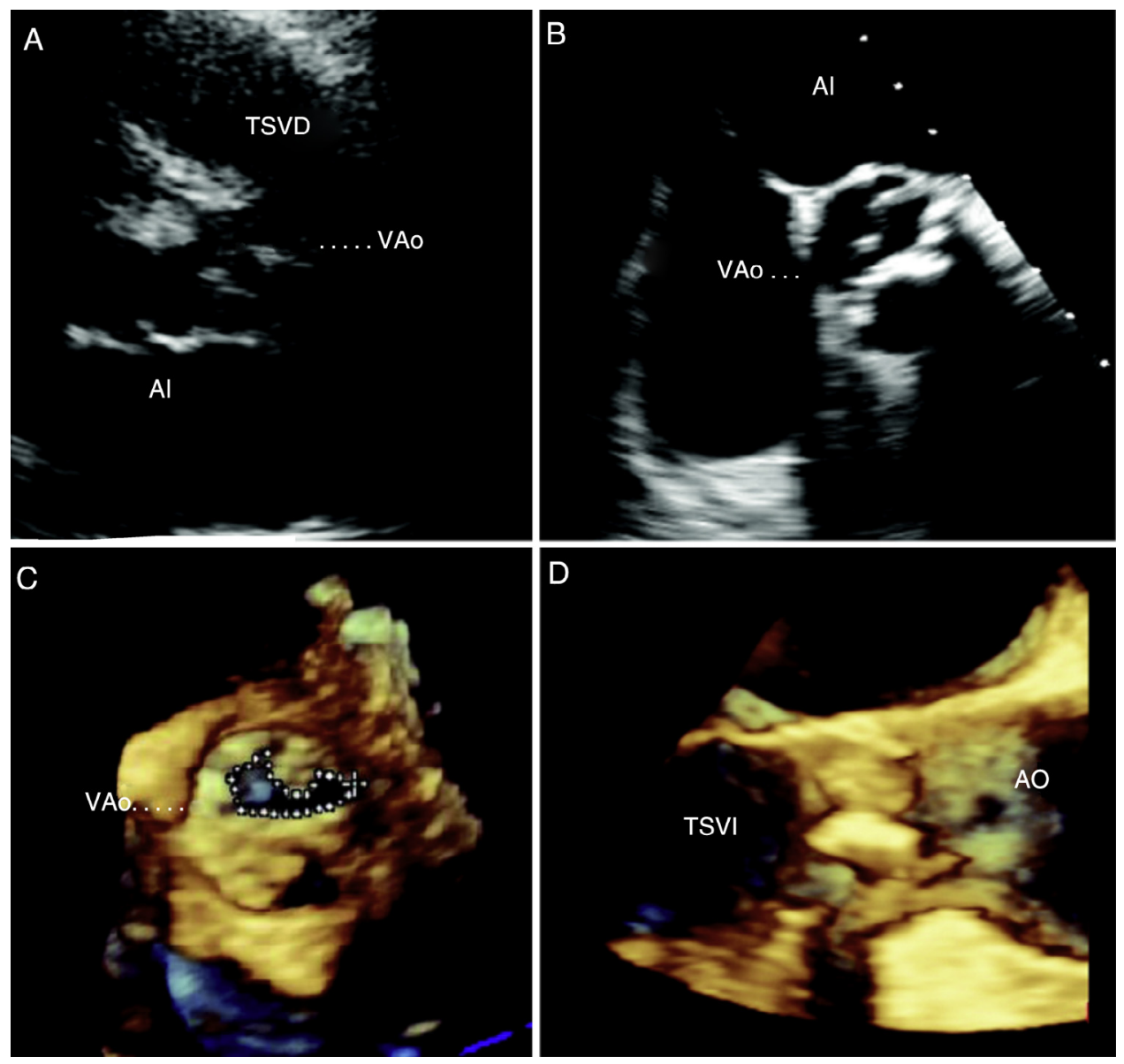

Figura 1 Imágenes ecocardiográficas de un hombre adulto mayor. a) Es la mejor imagen lograda por ecocardiografía transtorácica en la proyección del eje corto paraesternal y cuya calidad es deficiente. b) Mediante ecocardiograma transesofágico bidimensional se obtiene esta imagen en la posición medioesofágica con ajustes para captura transversal de la válvula aórtica. c) Imagen transesofágica tridimensional de la válvula aórtica en su eje transversal: obsérvense las marcas de planimetría; el área valvular fue de $0.70 \mathrm{~cm}^{2}$ (el gradiente transvalvular resultó en $54 \mathrm{mmHg}$ ). d) Imagen transesofágica tridimensional que muestra en eje longitudinal la salida del ventrículo izquierdo y la raíz aórtica y entre estas estructuras se ve la válvula aórtica deformada, muy engrosada y de aspecto fibrocalcífico. Al: aurícula izquierda; AO: aorta; TSVD: tracto de salida del ventrículo derecho; TSVI: tracto de salida del ventrículo izquierdo; VAo: válvula aórtica.

años de edad con xifosis y vértigo postural paroxístico que muestran los ajustes efectuados para obtener una posición corporal funcional y cómoda. En casos de espondiloartrosis cervical, entidad que suele ser dolorosa particularmente con los movimientos, debe considerarse la adopción de una posición antálgica compatible con el procedimiento procurando minimizar los movimientos del cuello mediante la asistencia de algún miembro del equipo que sujete la cabeza del paciente si fuera necesario. Cuando se trate de pacientes con lesiones articulares o fracturas óseas es prudente consultar con el especialista correspondiente qué movimientos, desplazamientos o posiciones evitar y cuáles preferir para elegir la más compatible con el procedimiento. Al no existir una recomendación universal sobre la posición del paciente en las diversas condiciones musculoesqueléticas, habrá de valorarse una estrategia particular para cada caso; el propósito fundamental es efectuar el procedimiento de ETE de la manera más funcional posible tanto para el paciente como para el examinador ya que trabajar en condiciones no ergonómicas puede afectar la calidad y la seguridad del procedimiento. Siempre será conveniente preguntar acerca de la existencia de trastornos de la micción, especialmente frecuentes en el octogenario varón con hiperplasia prostática, de manera que se tengan previstas algunas medidas como invitarle a vaciar su vejiga (de preferencia, y si es posible, en la comodidad del WC del gabinete) antes de comenzar el procedimiento y además sugerirle alguna seña o ademán que el paciente pueda hacer durante el estudio en caso de que perciba urgencia miccional, especialmente en pacientes conscientes en los que no se utilizó sedación. Similar consideración debe plantearse también en el caso de la anciana con incontinencia urinaria, más aún si se tiene en cuenta que la tos y el reflejo nauseoso («gag») al manipular la sonda transesofágica pueden suponer un esfuerzo, que aun siendo poco intenso, podría provocar escape de orina. El antecedente de vértigo postural puede advertirnos acerca de qué posición evitar para no disparar un paroxismo de vértigo que podría complicar significativamente el bienestar 


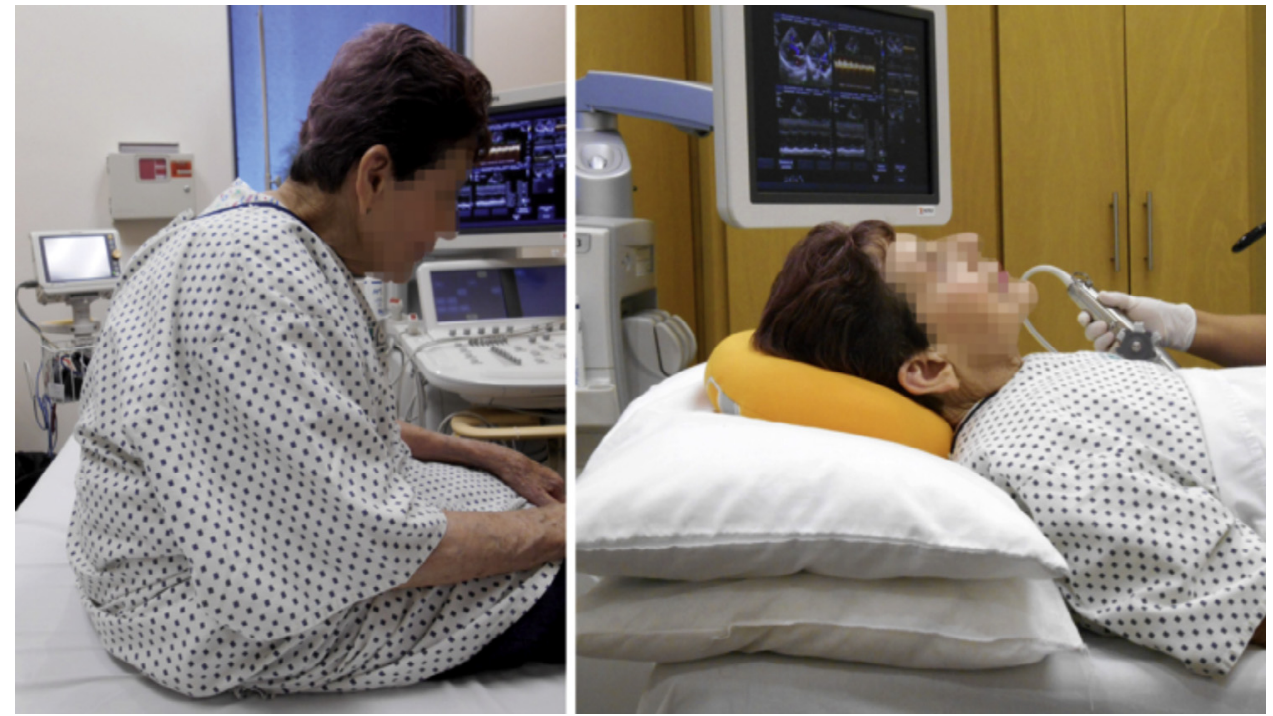

Figura 2 Imágenes de una paciente octogenaria con xifosis y vértigo postural. Obsérvense las adecuaciones efectuadas para mantener su cabeza en una elevación apropiada a sus condiciones, con el soporte de 2 almohadas y por encima de estas un cojín-dona de arena para mantener su cabeza cómodamente fija sin desviaciones laterales.

del anciano e incluso generarle un episodio nauseoso que abonará a la complejidad del procedimiento. Si se planea sedación es muy recomendable hacer un pausado proceso de idoneidad entre los medicamentos que habitualmente recibe el anciano y los fármacos sedantes que pudieran utilizarse durante el estudio a fin de advertir interacciones. Después del procedimiento, al considerar que el paciente ya está recuperado de la sedación, habrá que dedicarle especial atención a la primera ocasión en que se ponga de pie pues algunos fármacos de uso frecuente en el anciano como los antihipertensivos, antidepresivos, antiparkinsonianos y los alfabloqueadores utilizados para trastornos miccionales podrían sumar probabilidades de hipotensión ortostática sobre todo por interacción con efectos residuales de los sedantes $^{38}$. El anestesiólogo tendrá presente las recomendaciones personalizadas acerca de las dosis cautelosas de sedantes propuestas para ancianos, así como también tendrá en consideración la mayor probabilidad de hipotensión arterial en adultos mayores durante una ETE. Finalmente, los trastornos estructurales y funcionales del esófago, que son más frecuentes en el octogenario, habrán de considerarse en previsión del procedimiento a fin de tener advertencia sobre mayores cuidados en la introducción y desplazamientos de la sonda, e igualmente para la identificación oportuna de complicaciones que, aunque excepcionales, pudiesen ocurrir tras el estudio. En algún caso será prudente evitar el procedimiento y referir primero al paciente con el especialista digestivo, sobre todo en casos de disfagia u otros datos sospechosos de lesión esofágica.

En referencia a los hallazgos de alguna manera más peculiares en una ETE de un paciente octogenario, téngase presente una búsqueda más enfática de las condiciones patológicas anteriormente referidas como son la ateroesclerosis, el aneurisma y la disección de la aorta torácica, la calcificación valvular aórtica y mitral, y en general una búsqueda más esmerada de fuente embolígena. De igual manera procurar la identificación minuciosa de los elementos anatómicos y funcionales necesarios para la selección de pacientes que pudieran ser candidatos a procedimientos de intervención estructural.

\section{Conclusiones}

El adulto mayor, y en especial el paciente de 80 y más años de edad, debe considerarse perteneciente a una población vulnerable en relación con la realización de una ETE y que, en consecuencia, guarda unas características que le son peculiares. Tener estos elementos en consideración es fundamental para una mejor planeación del procedimiento así como para su misma realización, cuidados y vigilancia después del procedimiento. La mayor probabilidad de déficit cognitivo y de otras comorbilidades que son igualmente más frecuentes en el anciano merecen consideraciones especiales en relación con la calidad y la seguridad del procedimiento y de igual manera merece consideración especial la expectativa de ciertos hallazgos patológicos y diagnósticos más comunes en la edad avanzada. Así pues, las peculiaridades de la ETE en el octogenario hacen que esta modalidad diagnóstica implique diferencias con relación a pacientes de menor edad. Tales diferencias o peculiaridades deben ser conocidas y consideradas no solamente por quien efectúa el procedimiento sino de igual manera por el médico que refiere al paciente para el estudio -ciertamente con más frecuencia el cardiólogo pero también el cirujano cardiovascular, el neurólogo, el internista, el geriatra, el intensivista, el urgenciólogo y el infectólogo, entre otrosmás aún si se tiene en cuenta la hoy creciente afluencia de pacientes adultos mayores a los gabinetes de ETE.

\section{Responsabilidades éticas}

Protección de personas y animales. Los autores declaran que para esta investigación no se han realizado experimentos en seres humanos ni en animales. 
Confidencialidad de los datos. Los autores declaran que han seguido los protocolos de su centro de trabajo sobre la publicación de datos de pacientes.

Derecho a la privacidad y consentimiento informado. Los autores han obtenido el consentimiento informado de los pacientes y/o sujetos referidos en el artículo. Este documento obra en poder del autor de correspondencia.

\section{Financiación}

Ninguna.

\section{Conflicto de intereses}

Los autores declaran no tener ningún conflicto de intereses.

\section{Agradecimientos}

Agradecemos a la Lic. Enf. Gabriela Ruiz Villegas sus aportaciones tras la revisión del manuscrito y al Sr. Gustavo A. Noriega su valiosa contribución en el diseño gráfico.

\section{Bibliografía}

1. Corrada M, Brookmeyer R, Paganini-Hill A, et al. Dementia incidence continues to increase with age in the oldest old: The 90+ study. Ann Neurol. 2010;67:114-21.

2. Bermejo-Pareja F, Benito-León J, Vega S, et al. Incidence and subtypes of dementia in three elderly populations of central Spain. J Neurol Sci. 2008;264:63-72.

3. Evaluating prescription drugs used to treat: Alzheimer's disease comparing effectiveness, safety, and price. Consumer Reports Health. May, 2012.

4. Prince M, Bryce R, Albanese E, et al. The global prevalence of dementia: A systematic review and metaanalysis. Alzheimers Dement. 2013;9:63-7.

5. Loh KY, Ogle J. Age related visual impairement in the elderly. Med J Malasya. 2004;59:562-9.

6. Huang Q, Tang J. Age-related hearing loss or presbycusis. Eur Arch ORL. 2010;267:1179-91.

7. Jonsson R, Sixt E, Landahl S, et al. Prevalence of dizziness and vertigo in an urban elderly population. J Vestib Res. 2004; 14:47-52.

8. Katzman W, Wanej L, Shepherd J, et al. Age-related hyperkyphosis: Its causes, consequences, and management. J Orthop Sports Phys Ther. 2010;40:352-60.

9. Bentler SE, Liu L, Obrizan M, et al. The aftermath of hip fracture: Discharge placement, functional status change, and mortality. Am J Epidemiol. 2009;170:1290-9.

10. Lai B, Tsui J. Epidemiology of Parkinson's disease. BC Med J. 2001;43:133-7.

11. Van den Eeden S, Tanner C, Bernstein AL, et al. Incidence of parkinson's disease: Variation by age, gender, and race/ethnicity. Am J Epidemiol. 2003;157:1015-22.

12. Pringsheim T, Jette N, Frolkis A, et al. The prevalence of Parkinson's disease: A systematic review and meta-analysis. Mov Discord. 2014;29:1583-90.

13. Verhamme KM, Dieleman JP, Bleumink GS, et al. Incidence and prevalence of lower urinary tract symptoms suggestive of benign prostatic hyperplasia in primary care-the Triumph Project. Eur Urol. 2002;42:323-8.
14. Nitti V. The prevalence of urinary incontinence. Rev Urol. 2001;3 Suppl 1:S2-6.

15. Simóna MA, Bordasb JM, Campoc R, et al. Documento de consenso de la Asociación Española de Gastroenteología sobre sedoanalgesia en la endoscopia digestiva. Gastroenterol Hepatol. 2006;29:131-49.

16. Manika K, Navickas R, Nicol ED, et al. Safe combined intravenous opiate/benzodiazepine sedation for transoesophageal echocardiography. Br J Cardiol. 2010;17:125-7.

17. Stoddard MF, Longaker RA. The safety of transesophageal echocardiography in the elderly. Am Heart J. 1993;125:1358-62.

18. Scott H, Greenstein MD, David H, et al. Systemic atropine and glaucoma. Bull NY Acad Med. 1984;60:961-8.

19. Mandak JS, Minerva P, Wilson TW, et al. Angle closure glaucoma complicating systemic atropine use in the cardiac catheterization laboratory. Cathet Cardiovasc Diagn. 1996;39: 262-4.

20. Baldwin KM, Herbella F, Patti M, Espat NJ. Esophageal cancer. Medscape [Internet] [consultado 10 Ago 2015]. Disponible en: http://misc.medscape.com/pi/iphone/medscapeapp/html/ A277930-business.html

21. Kharasch D, Murali Sivarajan MB. Gastroesophageal perforation after intraoperative transesophageal echocardiography. Anesthesiology. 1996;85:426-8.

22. Clouse RE, Abramson BK, Todorczuk JR. Achalasia in the elderly. Effects of aging on clinical presentation and outcome. Dig Dis Sci. 1991;36:225-8

23. Canale A, Alvarez G, Cuen RE, et al. Prevalencia de polifarmacia en adultos mayores residentes de estancias de Hermosillo, Sonora. Epistemus. 2014;17:32-40.

24. Chugh SS, Blackshear JL, Shen WK, et al. Epidemiology and natural history of atrial fibrillation: Clinical implications. J Am Coll Cardiol. 2001;37:371-8.

25. Gómez-Doblas JJ, Muñiz J, Alonso Martín JJ, et al. Prevalencia de la fibrilación auricular en España. Resultados del estudio OFRECE. Rev Esp Cardiol. 2014;67:259-69.

26. Pujadas R, Arboix A, Anguera N, et al. Papel de las placas complejas de ateroma aórtico en la recurrencia del infarto cerebral de etiología incierta. Rev Esp Cardiol. 2005;58:34-40.

27. Dávila-Román VG, Barzilai B, Wareing TH, et al. Atherosclerosis of the ascending aorta. Prevalence and role as an independent predictor of cerebrovascular events in cardiac patients. Stroke. 1994;25:2010-6.

28. Adler Y, Fink N, Spector D, et al. Mitral annulus calcification: A window to diffuse atherosclerosis of the vascular system. Atherosclerosis. 2001;155:1-8.

29. Benjamin EJ, Plehn JF, D’Agostino RB, et al. Mitral annular calcification and the risk of stroke in an elderly cohort. $N$ Eng $J$ Med. 1992;327:374-9.

30. Davies MJ, Treasure T, Parker DJ. Demographic characteristics of patients undergoing aortic valve replacement for stenosis: Relation to valve morphology. Heart. 1996;75:174-8.

31. Pepi M, Evangelista A, Nihoyannopoulos P, et al. Recommendations for echocardiography use in the diagnosis and management of cardiac sources of embolism: European Association of Echocardiography (EAE) (a registered branch of the ESC). Eur J Echocardiogr. 2010;11:461-76.

32. Clouse WD, Hallett JW Jr, Schaff HV, et al. Improved prognosis of thoracic aneurysms: A population-based study. JAMA. 1998;280:1926-9.

33. Gilard M, Eltchaninoff $\mathrm{H}$, lung $B$, et al. Registry of transcatheter aortic-valve implantation in high-risk patients. N Eng J Med. 2012;366:1705-15.

34. Freixa X, Gafoor S, Regueiro A, et al. Comparison of efficacy and safety of left atrial appendage occlusion in patients aged $<75$ to $\geq 75$ years. Am J Cardiol. 2016;117:84-90.

35. Draper R, Colin T. Congenital heart disease in adults. Patient [Internet]. 2015 [consultado 13 Abr 2016]. Disponible en: 
http://patient.info/doctor/Congenital-Heart-Disease-inAdults.htm

36. Afilalo J, Therrien J, Pilote L, et al. Geriatric congenital heart disease: Burden of disease and predictors of mortality. J Am Coll Cardiol. 2011;58:1509-15.
37. Villarejo A, Puertas-Martín V. Utilidad de los test breves en el cribado de demencia. Neurología. 2011;26:425-33.

38. Craig GM. Clinical presentation of orthostatic hypotension in the elderly. Postgrad Med J. 1994;70:638-42. 